\title{
Derivación vesico-amniótica temprana en un feto de 12 semanas con megavejiga
}

\author{
Juan Manuel Acuña MD*; Juan Carlos Sabogal MD**
}

\section{RESUMEN}

Reportamos un caso enviado a nuestro servicio de una paciente en su semana 12 de embarazo con diagnóstico de megavejiga y valvas uretrales posteriores. Se practicó amniocéntesis para cariotipo y punción de la megavejiga para descompresión. Se realizó seguimiento ecográfico y se efectuó una segunda punción una semana después, para drenar el contenido vesical. Una semana más tarde se insertó un catéter de derivación vesico-amniótica vía transcutánea. Se observó recuperación del líquido amniótico y desapareció la dilatación del árbol urinario. A las 20 semanas, se observó desplazamiento del catéter, persistiendo la remisión del cuadro hasta el término de la gestación. Se realizó cesárea a la semana 36 y se obtuvo un neonato sano. La evaluación nconatal no encontró obstrucción anatómica en el tracto urinario. Hubo disfunción vesical que obligó al uso de una sonda de cistostomía por dos meses debido a disfunción vesical. En el momento, el paciente tiene dos años, con desarrollo psicomotor normal. Concluimos que la derivación vesico-amniótica temprana mejoró el pronóstico y previno secuelas en el presente caso.

PALABRAS CLAVES: Megavejiga, Cirugía Fetal, Derivación vesico-amniótica y obstrucción urinaria fetal.

\section{SUMMARY}

We report a case of a woman sent to us at 12 wecks gestation with a fetal diagnosis of megabladder and posterior urethral valves by routine ultrasound examination. We performed amniocentesis and the first bladder puncture for drainage purposes and Karyotyping at 12 weeks counted from the LMP. Follow up of the fetus was done every other day and a second puncture for drainage was done one week later. After 7 days, we performed the insertion of a modified catheter creating a vesicoamniotic shunt of urine. Amniotic fluid improved and dilation of urinary tract relapsed. At 20 weeks, displacement of catheter was found. However the shunt persisted functional until term. A cesarean section was performed at 36 weeks and a male healthy fetus was obtained. Mild prune belly was seen. Pediatric evaluation found no anatomical defect within the urinary tract and spontancous voiding was seen through the urethra. At 20 months age, patient has a normal development. We feel early derivation of megabladder improved the fetal prognosis and prevented secuelae.

KEY WORDS: Megabladder, fetal surgery, vesicoamniotic shunt, and fetal urinary tract obstruction.

\section{Introducción}

La obstrucción del tracto urinario tiene una incidencia que puede variar dependiendo de los criterios elegidos para el diagnóstico. Una cifra de promedio es de uno en 5000 a 8000 niños varones (Williams et al, 1965 ). Los casos no tratados pueden incluso llegar a la muerte aunque se han descrito remisiones espontáneas. Recientemente, con el desarrollo de la terapia fetal se ha reportado la implantación transcutánea de un catéter de derivación vesicoamniótico a las $16-17$ semanas (Wilhem et al, 1991 ). A pesar de que existe controversia, el momento de la intervención es de particular relevancia

\footnotetext{
* Profesor Asociado,

** Profesor Asistente. Departamento de Ginecología y Obstetricia de la Universidad Nacional de Colombia. Unidad de Biología Reproductiva, Instituto Materno Infantil. Bogotá.
}

para planear la inserción del catéter. Sin embargo, hay consenso acerca de que la instauración temprana del shunt mejora importantemente el pronóstico fetal (MacMahon et al, 1995).

\section{Caso clínico}

Se trata de una paciente de 33 años, que nos fue enviada con un diagnóstico de "embarazo de 12 semanas y valvas uretrales", hallazgos encontrados en un ultrasonido obstétrico de rutina. La paciente se encontraba en su segunda gestación. La primera tuvo un curso y producto normales. Los antecedentes obstétricos y ginecológicos eran irrelevantes y no había historia familiar de malformaciones. El primer ultrasonido a la semana 12 mostró un feto con una dilatación de la vejiga que alcanzaba los $40 \mathrm{~mm}$ de diámetro, asociado a dilatación ureteral y de las pelvis renales bilateralmente. 
Había compresión torácica secundaria, oligoamnios leve y no se encontraron otras malformaciones asociadas (figura 1). Se practicó entonces una primera amniocéntesis realizándose punción de la megavejiga y recuperándose $10 \mathrm{ml}$ de orina que se envió al laboratorio. Adicionalmente, se extrajeron $10 \mathrm{ml}$ de líquido amniótico para cariotipo. El análisis de orina fetal mostró : $\mathrm{K}+=3.15 \mathrm{mEq} / \mathrm{L}, \mathrm{Na}+=91 \mathrm{mEq} / \mathrm{L}, \mathrm{Ph}=7.5$, Densidad $=1.020 \mathrm{mOsm} / \mathrm{L}$ y Glucosa $=170 \mathrm{mg} / \mathrm{dl}$. El cariotipo fue reportado como 46, XY. A pesar de la punción, en el control ecográfico a las 48 horas se evidenció nuevo llenamiento de la vejiga, hasta el grado de distensión previo a la intervención. Luego de una semana, se realizó una segunda amniocéntesis con punción de la megavejiga y se extrajeron $50 \mathrm{ml}$ de orina fetal, para descomprimir el tórax fetal. Como el catéter de Rodeck usado en estos casos resultaba demasiado voluminoso para ésta edad gestacional, se modificó un catéter de Cook con doble cola de cerdo, de $150 \mathrm{~mm}$ de longitud, $1 \mathrm{~mm}$ de diámetro y se diseñó un trocar para su inserción a partir de una aguja de biopsia para tejido 16G, tipo "true-cut" ( figuras 2A y 2B). Durante una

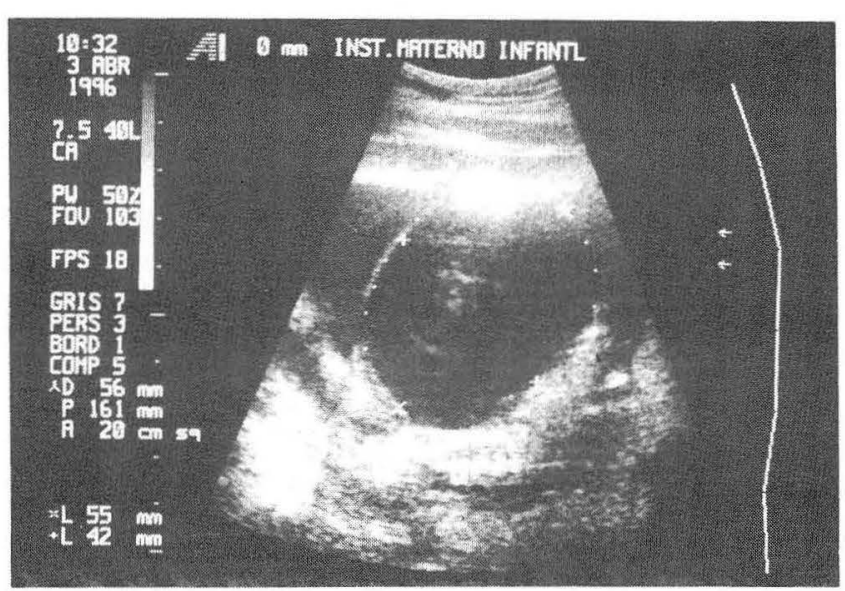

Figura 0004i01

Ultrasonido inicial. Se puede observar la vejiga fetal distendida y el cuerpo fetal remanente con el tórax, a la izquierda. Nótese la delgada pared de la vejiga y su contenido a tensión.

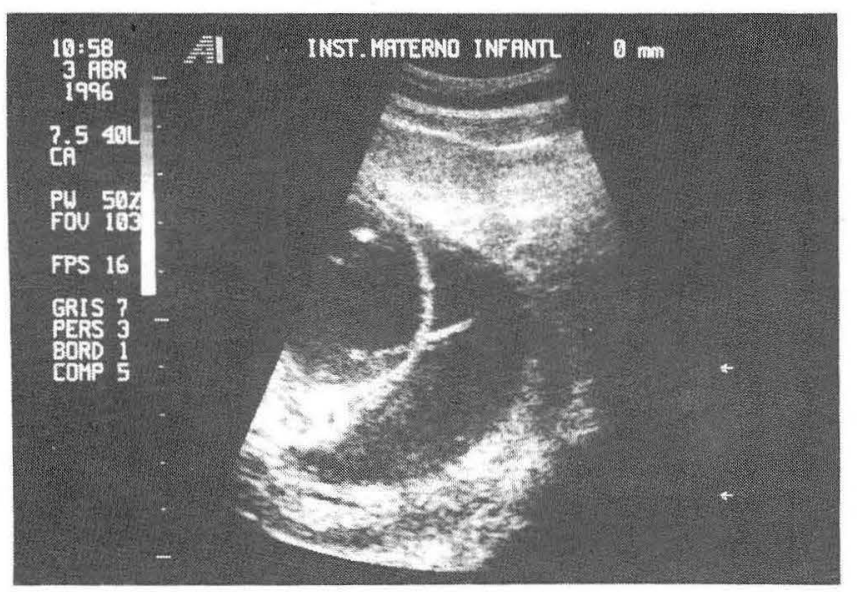

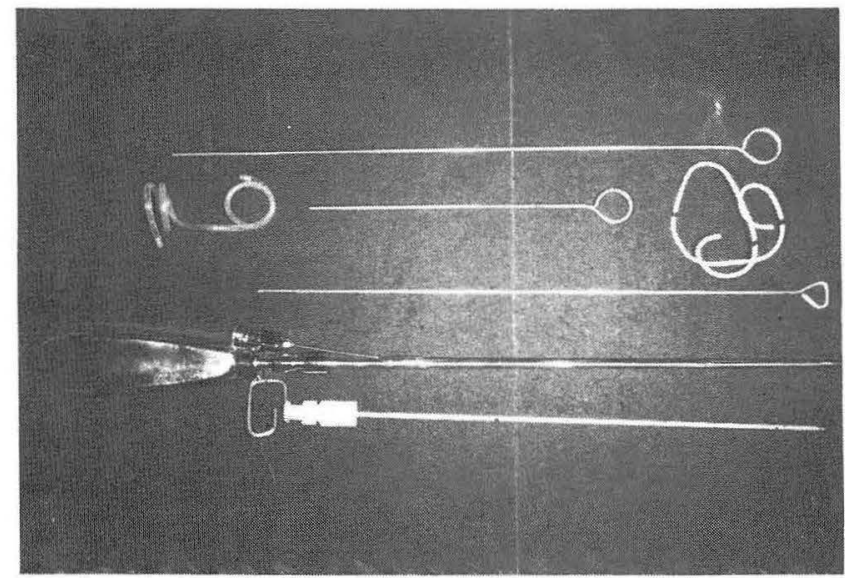

Figura $0004 \mathrm{i} 02$

Puede observarse el instrumental usado. En orden descendente, la aguja de "Tru-cut", el mandril usado, mandril accesorio, a la izquierda la modificación el catéter de Cook y a la derecha el catéter de Rodeck convencional, más voluminoso.

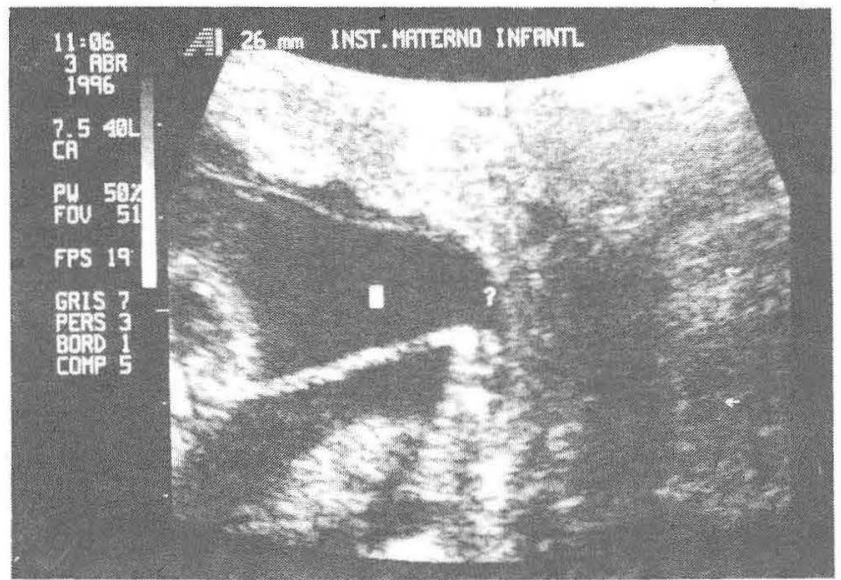

Figura 0004i03

A. Puede observarse el catéter en posición luego de la inserción. Un extremo dentro de la vejiga distendida, atravesando la pared y el otro extremo en el espacio amniótico, creando la derivación.

B. Se observa el anclaje del extremo proximal del catéter a la pared miometrial, que posteriormente fue liberado. 


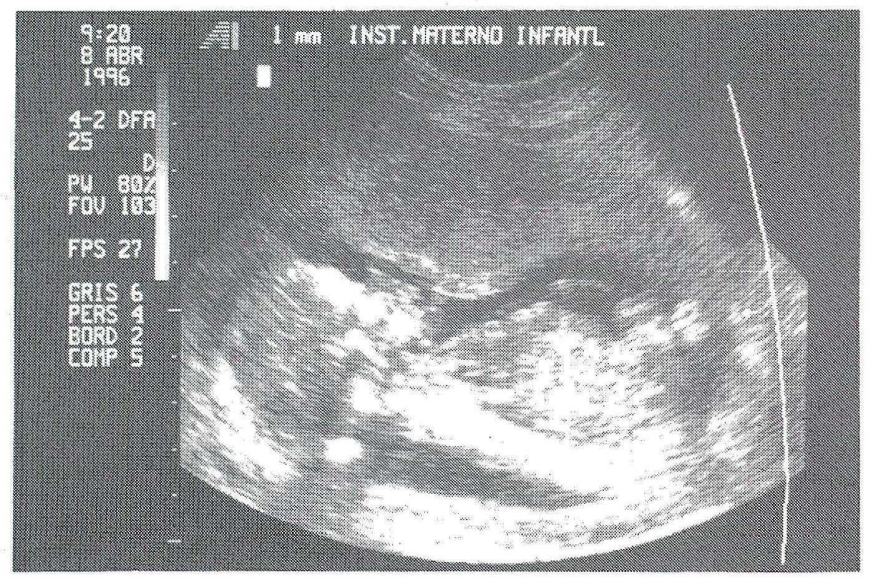

Figura $0004 \mathrm{i} 04$

A las 20 semanas, 6 semanas luego de la derivación, se observa un corte sagital de la anatomía fetal en que se observa retorno a la proporción normal entre tórax y abdomen. El cráneo y la cara fetales son fácilmente identificables a la izquierda de la imagen.

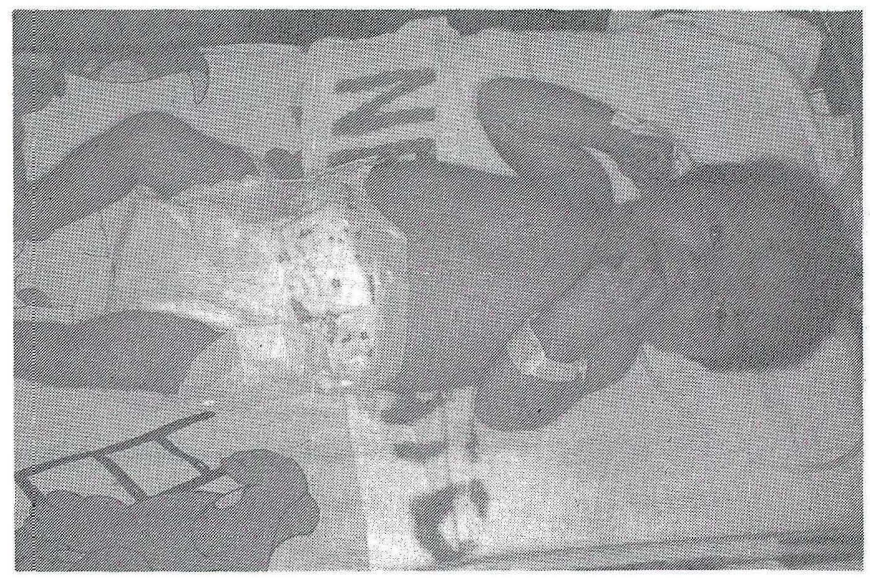

Figura 0004i05

Fotografía del neonato 48 horas luego del nacimiento. Nótese el aspecto normal del abdomen fetal.

desarrollo neurológico y pondoestatural adecuado. La función renal ha permanecido normal.

\section{Discusión}

Pensamos que el pronóstico fetal en el caso presentado mejoró de manera importante debido a la intervención fetal temprana con inserción del catéter, dado que no se presentaron secuelas a causa de la obstrucción urinaria. Los intentos previos de derivación de megavejiga reportados en la literatura se realizaron tan tempranamente como a la semana 17 (Wilhem et al, 1.991). Aun tardíamente en la gestación, parece justificarse la derivación vesico-amniótica, dados los reportes de que fetos de 24 (Nguyen et al, 1.996) y 31 (Cermak et al, 1.997) semanas han tenido mejor pronóstico que aquellos no tratados. Los estudios de electrolitos en la orina fetal realizados en el presente caso, revelaron una función renal intacta en el momento de la derivación vesico-amniótica lo cual seguramente explica el excelente pronóstico de este feto. De hecho, se ha reportado mejoría de parámetros hemodinámicos luego de la derivación (Tomlinson et al, 1997).

Es posible que la derivación vesico-amniótica temprana ofrezca dificultad técnica, dado que los catéteres disponibles para tal fin son demasiado grandes para ser insertados. Por tal razón vimos necesario diseñar un catéter de silástic más pequeño, a partir de un modelo estándar de Cook, èl cual fue insertado en una aguja 16G de "true-cut" usada comúnmente para biopsia de seno. Los extremos del catéter fueron curvados por medio de calor, sin alterar su forma hueca. La aguja de inserción se adhirió a un mango plástico proximal por medio de resinas epóxicas (figura 2). El anclaje durante la inserción fue causada por movimientos de la madre en el 


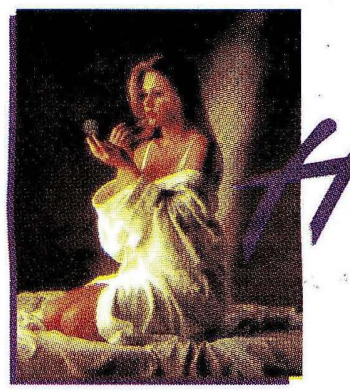

FORMULA

Cada gragea contiene: Gestodeno $75 \mathrm{mcg}$, Etinilestradiol $20 \mathrm{mcg}$, Lactosa 37,155 mg, azúcar 19,66 mg almidón de maiz $15,5 \mathrm{mg}$, carbonato de calcio $8,697 \mathrm{mg}$, talco $4,242 \mathrm{mg}$ polietilenglicol $60002,18 \mathrm{mg}$, povidona K-25 1,7 $\mathrm{mg}$, estearato de magnesio $550 \mathrm{mcg}$, povidona K-90 $171 \mathrm{mcg}$, cera E $50 \mathrm{mcg}$.

\section{INDICACIONES}

Harmonet está indicado para la prevención del embarazo en mujeres que deciden utilizar este método.

\section{POSOLOGIA Y FORMA DE ADMINISTRACION}

Para lograr una eficacia anticonceptiva óptima, HARMONET deberá ingerirse diariamente según las indicaciones y con intervalos diarios que no excedan las 24 horas. Debe instruirse a la paciente para que tome las grageas todos los días a la misma hora, preferiblemente con la comida de la noche o bien antes de acostarse.

\section{CONTRAINDICACIONES}

Harmonet está contraindicado en las mujeres que presenten:

Antedentes o diagnóstico de trastornos trombóticos arteriales o venosos, trastornos embólicos o situaciones que pudieran predisponer a la paciente (por ejemplo, defectos de la coagulación, valvulopatías cardiacas, fibrilación auricular); transtornos agudos o crónicos de la función hepática (incluyéndose entre éstos el síndrome de Dubin. diopatica o de prurito importante durante el embarazo; antecedentes o diagnóstico conocido o sospechado de lipíliasias hormonodependientes ( por ejemplo, cancer de mama o de endometrio); trastornos del metabolismo lipidico, anemia falciforme; diabetes mellitus con alteraciones vasculares; antecedentes de herpes gestacional sospechado; hipersensibilidad a cualquiera de los componentes de Harmonet.

\section{EFECTOS SECUNDARIOS}

Los siguientes efectos secundarios se han registrado en un total de 1338 pacientes que recibieron Harmonet durante el curso de tres estudios clínicos:

Generales Incidencia $>3 \%$ : dolor abdominal, dolor de espalda, dolor en los genitales, dolor pélvico. Incidencia < que 1\%: distensión del abdomen, abcesos, reacciones alérgicas, astenia, fiebre, síndrome gripal, pesadez en las extremidades, infecciones, malestar, moniliasis, artritis reumatoidea. Sistema Cardiovascula Incidencia $>3 \%$ : migraña. Incidencia de 1\%-3\%: venas varicosas. Incidencia $<1 \%$ : dolor al pecho trombosis de las venas profundas sofocos, hipertensión, palpitaciones, taquicardia, tromboembolia, tromboflebitis, vasodilatación, y otros transtornos cardiovasculares. Sistema Digestivo Incidencia <3\%: Náuseas. Incidencia 1\%-3\%: Vómito. Incidencia < $\%$ : colecistis, diarrea, flatulencia, transtornos vesiculares, gastritis, gastroenteritis, transtornos gastrointestinales, hepatopatia, aumento del apetito estomatitis. Sistema Metabólico Incidencia < $\%$ edema aumento o pérdida del peso. Sistema Nervioso Incidencia $>3 \%$ : Cefaleas, nerviosismo. Incidencia 1\%-3\%: Depresión, mareos, alteraciones en la libido. Incidencia $<1 \%$ : amnesia, ataxia, hostilidad, parestesia, transtornos en el sueño, somnolencia, sudoración excesiva. Sistema Respiratorio Incidencia <1\%: bronquitis, faringitis, rinitis, sinusitis. Dermatológicos. Incidencia > $3 \%$ : Acné. Indicidencia < 1\%: alopecía, cloasma, eczema, prurito, eritema, otros trastornos dermatológicos. Organos de los sentidos. Incidencia < $1 \%$ : visión anormal, tinnitus, sordera total transitoria. Sistema Urogenital. Incidencia $>3 \%$ : amenorrea, sangrando intermestrual, goteo, tensión mamaria. Incidencia < $1 \%$ : cistitis, dismenorrea, disuria, dolor en los genitales, galactorrea, leucorrea, netritis, quistes ováricos, cálculos renales infecciones del trato urinario, sequedad vaginal, moniliasis vaginal, vaginitis, transtornos vulvovaginales. Por otra parte, los siguientes efectos secundarios se han registrado en pacientes que recibian anticonceptivos orales y se consideraron relacionados con la droga administrada:Náuseas, Vómitos, síntomas gastrointestinales, (Tales como cólicos abdominales e inflamación), sangrado intermenstrual, goteo, alteraciones en el flujo menstrual, amenorrea, infertilidad temporaria con posterioridad a la descontinuación del tratamiento, edema, melasma que puede persistir, aumento o disminución de peso, alteraciones en la erosión y secreción cervical, disminución en el volumen de leche en el postparto inmediato, ictericia colestática, migraña, eritema (alérgico), depresión mental, menor tolerancia a los carbohidratos, candidiasis vaginal, acentuación de la curvatura de la córnea, intolerancia a los lentes de contacto, cambio en las mamas: tensión, aumento de tamaño, secreción. Existen evidencias de una posible relación entre los siguientes efectos secundarios y la utilización de anticonceptivos orales, aunque aún debe confirmarse esta información: Trombosis mesentérica, Trombosis retiniana. Los siguientes efectos secundarios se han registrado en pacientes que recibian anticonceptivos orales, pero $\mathrm{SU}$ asociación no ha sido aún confirmada ni rechazada: Anomalias congenitas, Sindrome premestrual, cataratas, neuritis óptica, cambios en el apetito, sindrome similar a la cistitis, cefaleas, nerviosismo, mareos, hirsutismo, pérdida del cabello, eritema multiforme, eritema nodoso, erupción hemorrágica, vaginitis, disminución de la función renal, síndrome urémico hemolitico, síndrome de BuddChiari, acné, cambios en la líbido. colitis, enfermedad cerebrovascular, con prolapso de la válvula mitral, sindrome similares a lupus.

Para mayor información, véanse las advertencias y precauciones.

\section{SOBREDOSIS}

La sobredosis puede causar náuseas o vómitos; en las mujeres puede producirse sangrado por supresión. En niños, no se han registrado efectos graves después de la utilización de altas dosis de anticonceptivos orales.

\section{PRESENTACION}

Envase con 21 grageas. Registro INVIMA 006606 momento de retirar el catéter de la aguja de inserción y su trocar.

Finalmente, el resultado de este caso probablemente se correlaciona con la inserción temprana de la derivación vesico-amniótica en un momento en el cual la función renal aún estaba indemne. A pesar de que son necesarios más estudios a fin de precisar sus indicaciones y el momento adecuado, consideramos probable que la derivación vesico-amniótica temprana sea un procedimiento seguro con un catéter más pequeño, que podría mejorar el pronóstico fetal en casos seleccionados de obstrucción del tracto urinario, con megavejiga.

\section{BIBLIOGRAFIA}

1. Cermak A., Vlasin P., Pacik D. Prenatal use of a vesico amniotic shunt in obstructive uropathy. Rozhl Chir, 1997; 76(1): 6-8.

2. MacMahon RA., Renou PM., Shekleton PA., Paterson PJ. Severe urethral obstruction diagnosed at 14 weeks gestation: variability of outcome with and without drainage. Fetal Diagn Ther, 1995; 10(5): 343-348.

3. Nguyen Th., Thorup JM., Larsen T. Vesicoamniotic shunt therapy in fetal obstructive uropathy. Ugeskr Laeger, 1996; 158(39): 54635464 .

4. Tomlinson MW., Johnson MP., Gonclaves L., King M., Freedman A., Smith C., Hume RF Jr, Evans MI. Correction of hemodynamic abnormalities by vesicoamniotic shunting in familial congenital megacystis. Fetal Diagn Ther, 1996; 11(1): 46-49.

5. Wilhem C., Wieacker P., Quaas L., Schillinger H. Fetal urinary tract obstructions: Prenatal diagnosis. Prenatal and postnatal therapy. J Perinat Med, 1991; 19(5): 357-65.

6. Williams DI., Eckstein HB. Obstructive valves in the posterior urethra. J Urol, 1965; 93: 236.

\section{Wyeth}

Lider mundial en el cuidado de la salud femenina 